Vietnam Journal of Mechanics, VAST, Vol.37, No. 2 (2015), pp. 81 - 90

DOI:10.15625/0866-7136/37/2/5019

\title{
A CELL-BASED SMOOTHED DISCRETE SHEAR GAP METHOD (CS-FEM-DSG3) FOR DYNAMIC RESPONSE OF LAMINATED COMPOSITE PLATE SUBJECTED TO BLAST LOADING
}

\author{
Dang Trung Hau, Nguyen Thoi My Hanh, Nguyen Thoi Trung* \\ Ton Duc Thang University, Ho Chi Minh City, Vietnam \\ *E-mail: nguyenthoitrung@tdt.edu.vn \\ Received September 24, 2014
}

\begin{abstract}
The paper investigates the dynamic response of laminated composite plate under the effect of blast loading. The cell-based smoothed discrete shear gap method (CSFEM-DSG3) based on the first-order shear deformation theory (FSDT) and the equivalent layer theory (ELT) is used to model the behavior of the laminated composite plate. The blast loading is simulated by a time diagram of rectangular, triangular, exponential, or sinusoidal shape. The effects of the number of layers and fiber orientation to the displacement and stress fields of the laminated composite plate are discussed. Numerical results demonstrated the accuracy and reliability of the present method compared with previous published methods.
\end{abstract}

Keywords: Laminated composite plate, blast loading, cell-based smoothed discrete shear gap method (CS-FEM-DSG3), first-order shear deformation theory (FSDT), equivalent layer theory (ELT).

\section{INTRODUCTION}

Nowadays, laminated composite plate has become one of indispensable components in numerous engineering structures varying from automotive industry, civil infrastructure to aerospace structures. Owning to many superior properties such as high stiffness, light weight, long fatigue life, flexible fiber orientation, electric resistant, etc, the laminated composite plate is expected to sustain over many difficult situations. Among them, blast loading, an intensive high load happening in a very short time, is one of the most challenging situations that the laminated composite plate may undergo during its operation. Hence, analyzing the dynamic response of the laminated composite plate under the influence of blast loading is a necessary topic that can contribute important insights to researchers, and has attracted a number of research works in the field of numerical methods during the last decade. Some typical works can be listed as follows.

(C) 2015 Vietnam Academy of Science and Technology 
Khdeir and Reddy [1] used the method of the orthogonality of principal modes and the state variables technique to investigate the dynamic response of symmetric cross-ply laminated composite plates by solving the equation of a higher-order shear deformation plate theory (HSDT). Librescu and Nosier [2] dealt with a theoretical analysis of the dynamic response of shear deformable symmetrically laminated rectangular composite flat panels exposing to boom and explosive blast loadings. In this study, the time history of the sonic boom was described as an $N$-shaped pulse, whose negative phase duration was included as a variable in the analysis. The governing equation system formulated by HSDT was solved by using the integral-transform technique. Lam and Chun [3] considered the dynamic response of laminated angle-ply plate with the clamped boundary conditions subjected to explosive blast loading. The plate was modeled by classical plate theory (CPT) and the dynamic analysis was carried out by the method of normal mode superposition based on Rayleigh-Ritz method. Meimaris and Day [4] studied the dynamic response of laminated anisotropic plate which was modeled by the twenty nodes, isoparametric, parabolic, solid element. Satish Kumar and Mukhooadhyay [5] analyzed the transient response of laminated stiffened plates under step and air-blast loads. The formulation was based on the first order shear deformation theory (FSDT). Hause and Librescu [6] presented an analytical study of the dynamic response in bending of flat sandwich panels characterized by laminated face sheet and weak core. In this study, the adopted solution methodology was based on the extended Galerkin method coupling with the Laplace Transform to get closed-form solutions of the problem. Librescu et al. [7] analyzed the dynamic response of geometrically non-linear sandwich flat panels subjected to initial geometric imperfection and explosive blast loading produced by underwater and in-air explosions.

In general, the previous works mostly employed the HSDT theory or the analytical formulations to achieve good results. The approach using high-order formulas is computationally expensive, while the analytical approach is hard to extend to problems of arbitrary geometries or complicated boundary conditions. Hence, conducting new numerical methods that can deliver accurate results by means of simple implementations is an obvious demand. This paper aims to fill this gap by introducing a new method that combines advantages from many recently proposed methods derived from triangular finite elements.

In an advanced extent of numerical methods, Liu and Nguyen [8] have successfully developed robust and efficient series of methods termed the smoothed finite element methods (S-FEMs) that outperform the traditional finite elements in term of accuracy and efficiency. The S-FEMs consist of two key mechanisms: (1) they usually employ the three-node triangular elements for easy mesh generation on arbitrary geometries; (2) they integrate the strain smoothing technique in computing the local stiffness based on smoothing domains to soften the overly-stiffness of the ordinary triangular elements. Different variants of S-FEMs are distinguished based on the manners of creating the smoothing domains. Among members of S-FEMs, the cell-based smoothed finite element (CS-FEM) has been developed widely for structural analysis due to possessing many beneficial properties in analyzing static and dynamic response of 2D plate problems [9]. Recently, the CS-FEM was combined with the discrete shear gap method using 
triangular element (DSG3) [10] to give a so-called the cell-based smoothed discrete shear gap method (CS-FEM-DSG3) [11] which can help eliminate the shear locking phenomena and improve efficiently the accuracy and stability of the original DSG3 in analyzing plate and shell problems [11-16].

In this paper, the CS-FEM-DSG3 element is extended to analyze the behavior of laminated composite plate subjected to blast loading. A time function of blast loading may take a rectangular, triangular, exponential, or sinusoidal shape. Some numerical examples are performed to illustrate the accuracy and reliability of the proposed method. The numerical results will be compared with the previous published methods.

The paper consists of 5 sections, in which section 2 briefly introduces the mathematical formulation and section 3 presents the CS-FEM-DSG3. Section 4 illustrates the numerical results. Some discussion and conclusion are given in the last section.

\section{MATHEMATICAL FORMULATION OF LAMINATED COMPOSITE PLATE AND THE BLAST LOADING}

Let us consider a rectangular composite plate in the Cartesian coordinated $0 x y z$ in which the $x-y$ plane coincides with the middle plane $\Omega$ of the plate as shown in Fig. 1 .

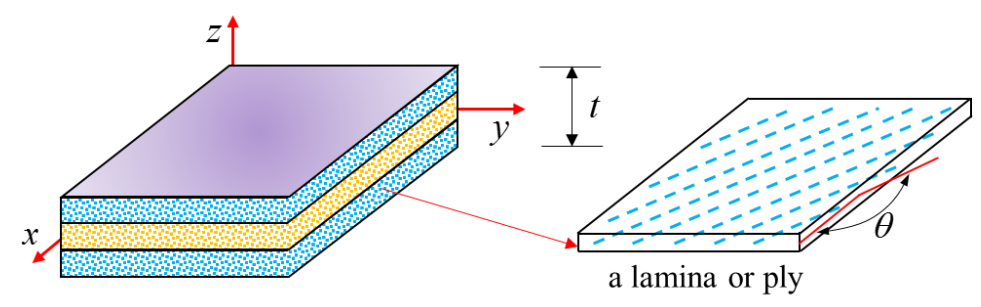

Fig. 1. Laminated composite plate

The plate consists of $N$ orthotropic cross-ply or angle-ply layers with a total thickness $t$. The displacement field at any point on the middle plane is given by

$$
\mathbf{u}=\left\{u_{0}, v_{0}, w_{0}, \beta_{x}, \beta_{y}\right\}^{T},
$$

where $u_{0}$ and $v_{0}$ are the membrane displacements along the $x$ and $y$-axis, respectively; $w_{0}$ is the transverse displacements along the $z$-axis; $\beta_{x}$ and $\beta_{y}$ are, respectively, the rotations of the transverse normal of the middle plane about the $y$ and $x$-axis.

According to FSDT theory $[17,18]$, the Galerkin weak form of the composite plate subjected to blast loading without damping system is given by

$$
\int_{\Omega}\left\{\delta\left(\boldsymbol{\varepsilon}_{m}\right)^{T} \delta(\boldsymbol{\kappa})^{T} \delta(\boldsymbol{\gamma})^{T}\right\}\left[\begin{array}{ccc}
\mathbf{D}_{m} & \mathbf{D}_{m b} & 0 \\
\mathbf{D}_{m b} & \mathbf{D}_{b} & 0 \\
0 & 0 & \mathbf{D}_{s}
\end{array}\right]\left\{\begin{array}{c}
\boldsymbol{\varepsilon}_{m} \\
\boldsymbol{\kappa} \\
\boldsymbol{\gamma}
\end{array}\right\} \mathrm{d} \Omega+\int_{\Omega} \delta(\mathbf{u})^{T} \operatorname{mü} \mathrm{d} \Omega=\int_{\Omega} \delta(\mathbf{u})^{T} \mathbf{b} \mathrm{d} \Omega,
$$


where $\boldsymbol{\varepsilon}_{m}, \boldsymbol{\kappa}$ and $\boldsymbol{\gamma}$ are the membrane, bending and shear strains of the plate, given by $\boldsymbol{\varepsilon}_{m}=\left\{\begin{array}{lll}u_{0, x} & v_{0, y} & u_{0, y}+v_{0, x}\end{array}\right\}^{T}, \boldsymbol{\kappa}=\left\{\begin{array}{lll}\beta_{x, x} & \beta_{y, y} & \beta_{x, y}+\beta_{y, x}\end{array}\right\}^{T}, \boldsymbol{\gamma}=\left\{w_{0, x}+\beta_{x} \quad w_{0, y}+\beta_{y}\right\}^{T}$.

In Eq. (2), $\mathbf{D}_{m}, \mathbf{D}_{m b}, \mathbf{D}_{b}$ and $\mathbf{D}_{s}$ are the material matrices of the composite plate, defined by

$$
\begin{aligned}
\left(\mathbf{D}_{m}, \mathbf{D}_{m b}, \mathbf{D}_{b}\right) & =\sum_{k=1}^{N} \int_{z_{k}}^{z_{k+1}}\left(\bar{Q}_{i j}\right)_{k}\left(1, z, z^{2}\right) \mathrm{d} z, \quad i, j=1,2,6, \\
\left(\mathbf{D}_{s}\right) & =\sum_{k=1}^{N} \int_{z_{k}}^{z_{k+1}} \kappa\left(\bar{Q}_{i j}\right)_{k} \mathrm{~d} z, \quad i, j=4,5,
\end{aligned}
$$

where $\kappa=5 / 6$ is shear coefficient, $\bar{Q}_{i j}$ are the transformed material constants of the $k$-th lamina [19], $\mathbf{m}$ is the mass matrix containing the mass density of the material $\rho$, expressed by

$$
\mathbf{m}=\sum_{k=1}^{N} \rho_{(k)} \int_{z_{k}}^{z_{k+1}}\left[\begin{array}{ccccc}
1 & 0 & 0 & z & 0 \\
0 & 1 & 0 & 0 & z \\
0 & 0 & 1 & 0 & 0 \\
z & 0 & 0 & z^{2} & 0 \\
0 & z & 0 & 0 & z^{2}
\end{array}\right] \mathrm{d} z
$$

$\mathbf{b}=\{0,0, q, 0,0\}^{T}$ is the distributed load vector, where $q$ is the sinusoidal distributed load, defined by

$$
q=Q \sin \left(\frac{\pi x}{a}\right) \sin \left(\frac{\pi y}{b}\right)
$$

in which, $\mathrm{Q}$ is the blast loading, defined by [1]

$$
Q=q_{0} F(t),
$$

where $q_{0}$ is the peak blast pressure and $F(t)$ is the time-dependent dynamic loading, given by

$$
F(t)=\left\{\begin{array}{lll}
\sin \left(\pi t / t_{p}\right) & \text { if } 0 \leq t \leq t_{p}, & \text { sine loading } \\
0 & \text { if } t \geq t_{p}, \\
1 & \text { if } 0 \leq t \leq t_{p}, & \text { step loading } \\
0 & \text { if } t \geq t_{p}, \\
1-t / t_{p} & \text { if } 0 \leq t \leq t_{p}, & \text { triangular loading } \\
0 & \text { if } t \geq t_{p}, & \text { exponential loading }
\end{array}\right.
$$

in which, $t_{p}$ is the positive phrase duration and $\psi$ is the waveform parameter.

Note that, the usage of the FSDT in the present research is due to the simplicity in the formulation of the CS-DSG3 integrated with the FSDT and the existence of available reference numerical results in Ref. [1] which also use the FSDT. 


\section{BRIEF ON CS-FEM-DSG3 FORMULATION}

In the CS-FEM-DSG3 [11], the domain discretization is the same as that of the standard FEM using $N_{n}$ nodes and $N_{e}$ triangular elements. However in the formulation of the CS-FEM-DSG3, each triangular element $\Omega_{e}$ is further divided into three sub-triangles $\Delta_{1}$, $\Delta_{2}$ and $\Delta_{3}$ by connecting the central point $\mathrm{O}$ of the element to three field nodes as shown in Fig. 2.

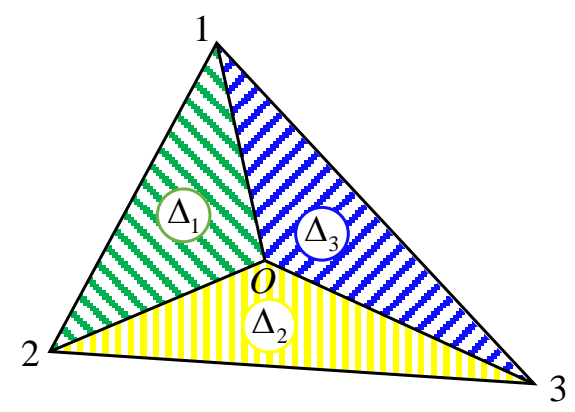

Fig. 2. Three sub-triangles $\left(\Delta_{1}, \Delta_{2}\right.$ and $\left.\Delta_{3}\right)$ created from the triangle 1-2-3 in the CS-FEM-DSG3 by connecting the central point $\mathrm{O}$ with three field nodes 1,2 and 3

Then in each sub-triangle $\Delta_{i}, i=1,2,3$, the strain fields are calculated by using the discrete shear gap method (DSG3) [10]. Finally, the strain fields of the element $\Omega_{e}$ are obtained by applying the cell-based strain smoothing operation in the CS-FEM [8]. Details of the formulation CS-FEM-DSG3 can be found in [11-13,15,16].

After the above mentioned process, the strain fields are re-approximated by

$$
\boldsymbol{\varepsilon}_{m}=\tilde{\mathbf{B}}_{m} \mathbf{d}_{e}, \quad \boldsymbol{\kappa}=\tilde{\mathbf{B}}_{b} \mathbf{d}_{e}, \quad \boldsymbol{\gamma}=\tilde{\mathbf{B}}_{s} \mathbf{d}_{e},
$$

in which, $\mathbf{d}_{e}=\left\{u_{i}, v_{i}, w_{i}, \beta_{x i}, \beta_{y i}\right\}^{T}, i=1,2,3$ is the displacement field of element $\Omega_{e}$; $\tilde{\mathbf{B}}_{m}, \tilde{\mathbf{B}}_{b}$ and $\tilde{\mathbf{B}}_{s}$ are, respectively, the smoothed membrane, bending and shear strain gradient matrices, defined by

$$
\tilde{\mathbf{B}}_{i}=\sum_{j=1}^{3} \frac{A_{\Delta_{j}} \mathbf{B}_{i}^{\Delta_{j}}}{A_{e}},
$$

where $A_{e}$ is the area of element $\Omega_{e} ; A_{\Delta_{j}}$ and $\mathbf{B}_{i}^{\Delta_{j}}$ are respectively the area and strain gradient matrices of sub-triangle $\Delta_{j}$, in which, $i=m, b, s$ represents the membrane, bending and shear components.

By substituting formula (9) into the weak form (2), the equilibrium equation for the plate subjected to blast loading without damping system is presented as

$$
\mathbf{M} \ddot{\mathbf{d}}_{t}+\mathbf{K} \mathbf{d}_{t}=\mathbf{F}_{t},
$$

where $\mathbf{M}$ and $\mathbf{K}$ are the global mass matrix and stiffness matrix, respectively, defined by

$$
\mathbf{M}=\sum_{e=1}^{N_{e}} \int_{\Omega_{e}} \mathbf{N}^{T} \mathbf{m} \mathbf{N d} \Omega,
$$




$$
\mathbf{K}=\sum_{e=1}^{N_{e}} \int_{\Omega_{e}}\left\{\begin{array}{ccc}
\tilde{\mathbf{B}}_{m}^{T} & \tilde{\mathbf{B}}_{b}^{T} & \tilde{\mathbf{B}}_{s}^{T}
\end{array}\right\}\left[\begin{array}{ccc}
\mathbf{D}_{m} & \mathbf{D}_{m b} & 0 \\
\mathbf{D}_{m b} & \mathbf{D}_{b} & 0 \\
0 & 0 & \mathbf{D}_{s}
\end{array}\right]\left\{\begin{array}{c}
\tilde{\mathbf{B}}_{m} \\
\tilde{\mathbf{B}}_{b} \\
\tilde{\mathbf{B}}_{s}
\end{array}\right\} \mathrm{d} \Omega .
$$

The displacement $\mathbf{d}$ and the acceleration $\ddot{\mathbf{d}}$ in Eq. (11) are obtained using Newmark method [20], in which, the displacement and the acceleration at the initial condition are expressed as

$$
\mathbf{d}_{t=0}=\dot{\mathbf{d}}_{t=0}=0 .
$$

At time step $t+1$, the acceleration is calculated based on the equation equilibrium as

$$
\left(\mathbf{M}+\mathbf{K} \beta \Delta t^{2}\right) \ddot{\mathbf{d}}_{t+1}=\mathbf{F}_{t+1}-\mathbf{K}\left(\mathbf{d}_{t}+\dot{\mathbf{d}}_{t} \Delta t+\ddot{\mathbf{d}}_{t}(1 / 2-\beta) \Delta t^{2}\right) .
$$

Then, the velocity and displacement at time step $t+1$ are updated via

$$
\begin{aligned}
& \dot{\mathbf{d}}_{t+1}=\dot{\mathbf{d}}_{t}+\ddot{\mathbf{d}}_{t}(1-\lambda) \Delta t+\ddot{\mathbf{d}}_{t+1} \lambda \Delta t, \\
& \mathbf{d}_{t+1}=\mathbf{d}_{t}+\dot{\mathbf{d}}_{t} \Delta t+\ddot{\mathbf{d}}_{t}(1 / 2-\beta) \Delta t^{2}+\ddot{\mathbf{d}}_{t+1} \beta \Delta t^{2},
\end{aligned}
$$

Since the value of $\omega$, natural frequency of the composite plate, is not considered in this paper, the average acceleration method (with $\lambda=1 / 2$ and $\beta=1 / 4$ as shown in Ref. [21]) which can ensure the stability condition is chosen in the paper.

Also note that the cell-based gradient smoothing technique is only applied for improving the stiffness matrix of the CS-FEM-DSG3, without being applied to the other matrix or vector (such as mass matrix or load vector). The computation of mass matrix and load vector is quite similar to the traditional way of the standard FEM.

\section{NUMERICAL RESULTS}

In this section, the dynamic response of a simply supported square laminated composite plate subjected to blast loading is investigated in 0.008 seconds with increment of 0.000016 seconds. The model of the blast loading is performed by repeating accurately the model of the blast loading in Ref. [1] to make sure that the comparison between results is consistent. The plate is composed of three cross-ply $\left(0^{\circ} / 90^{\circ} / 0^{\circ}\right)$ layers with identical material parameters such as: Young's modulus $E_{1}=25 \times 10^{6} \mathrm{psi}, E_{2}=10^{6} \mathrm{psi}$; shear's modulus $G_{12}=G_{13}=0.5 \times 10^{6}$ psi, $G_{23}=0.2 \times 10^{6}$ psi; Poisson ratio $v_{12}=0.25$; and mass density $\rho=0.05791 \mathrm{lb} / \mathrm{in}^{3}$. The thickness of layers are assumed to be the same and the total thickness of the laminate is $h=6 \mathrm{in}$. The size of the plate is $a=30 \mathrm{in}$. The plate is subjected to sinusoidally distributed pressure with the peak value $q_{0}=10^{4} \mathrm{psi}$. The constant coefficients in Eq. (8) are chosen with $t_{p}=0.006 \mathrm{~s}$ and $\gamma=330 \mathrm{~s}^{-1}$.

Fig. 3 shows the central deflection as a function of time corresponding to different pulse loadings by the CS-FEM-DSG3, DSG3 (meshing $12 \times 12 \times 2$ triangular elements) and previous published results. It is seen that the results by the CS-FEM-DSG3 agree well with those from Khdeir and Reddy [1] using analytical solution based on the FSDT theory, and outperform those by the DSG3. This is because the cell-based gradient smoothing technique of the CS-FEM-DSG3 helps soften the over-stiffness of the DSG3 as shown in Ref. [11], and hence make the results by the CS-FEM-DSG3 more accurate than those by the DSG3. 


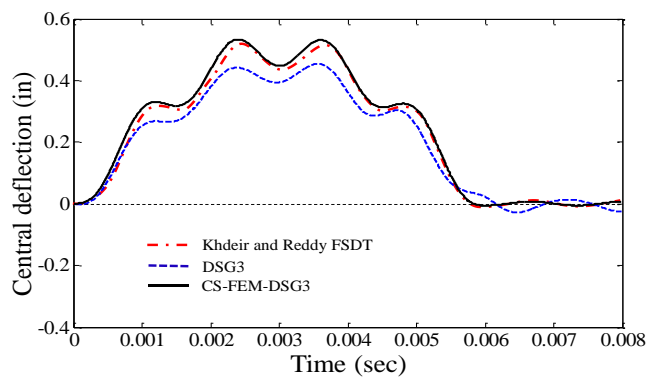

(a)

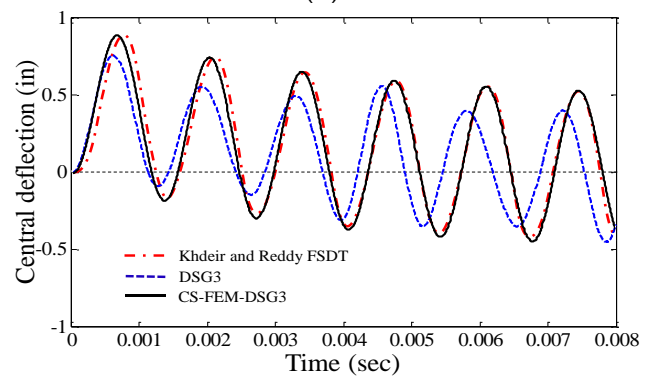

(c)

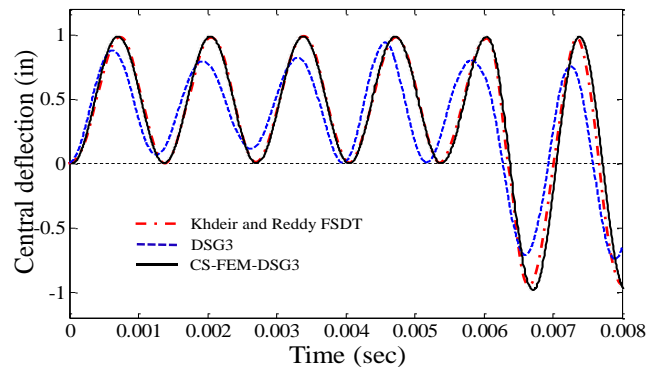

(b)

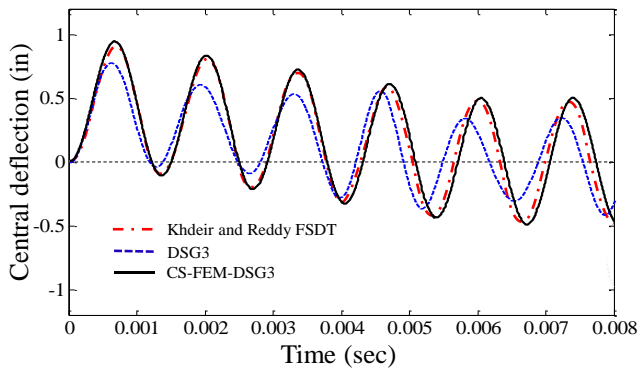

(d)

Fig. 3. Variation of the center deflection as a function of time for various pulses loading: a) sine; b) step; c) exponential; d) triangle

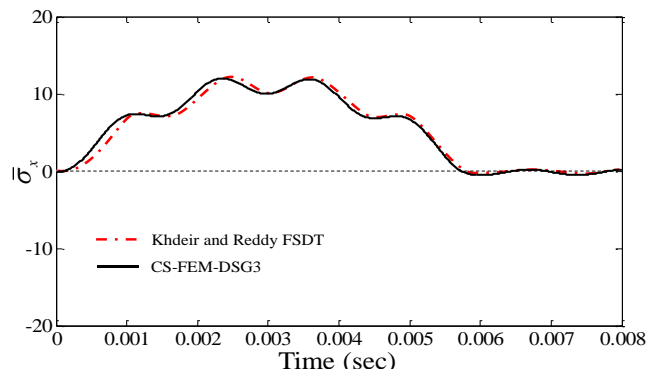

(a)

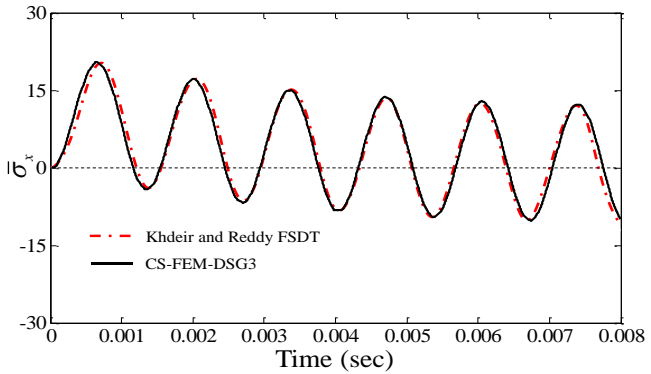

(c)

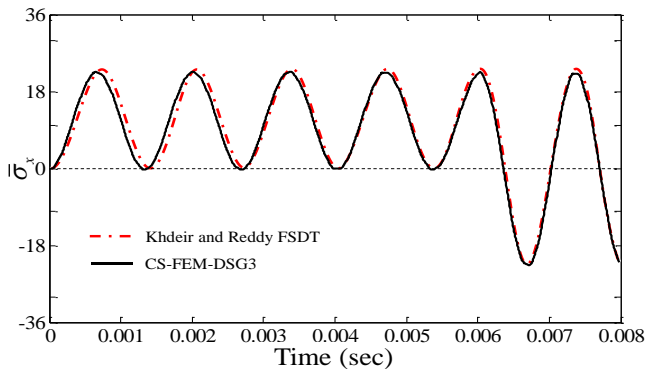

(b)

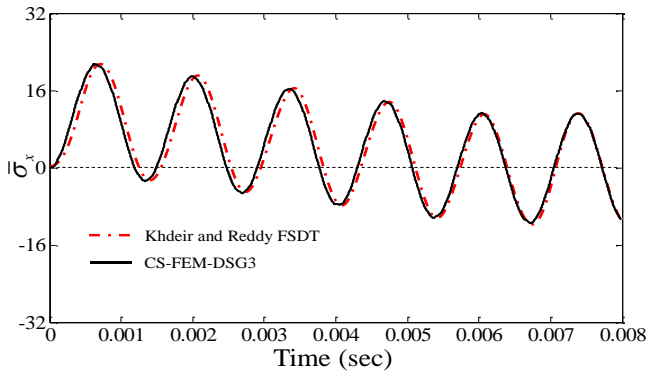

(d)

Fig. 4. Variation of the normal stress $\bar{\sigma}_{x}=\sigma_{x}(a / 2, a / 2, h / 2) / q_{0}$ as a function of time for various pulse loadings: a) sine; b) step; c) exponential; d) triangle 
Similarly, Fig. 4 presents the normal stress as a function of time for the various pulse loadings, obtained by the CS-FEM-DSG3 and by Khdeir and Reddy [1]. The two results match perfectly. This hence illustrates again the accuracy and robustness of the present method.

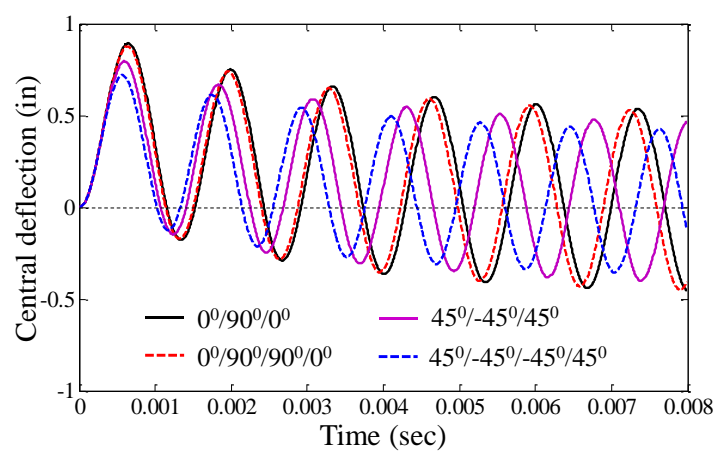

(a)

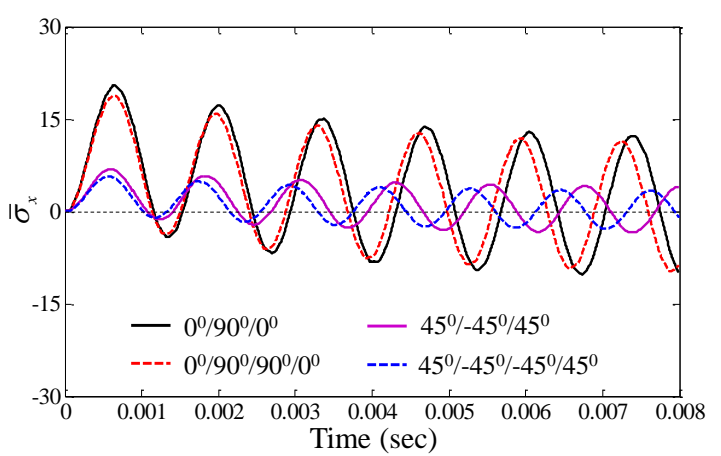

(b)

Fig. 5. Central deflection and normal stress of the plate by the CS-FEM-DSG3 (as a function of time for exponential pulse loading) subjected to various number of layers and fiber orientations;

(a) Central deflection (in) and b) Normal stress $\bar{\sigma}_{x}=\sigma_{x}(a / 2, a / 2, h / 2) / q_{0}$

Fig. 5 illustrates the central deflection and normal stress of the plate by the CSFEM-DSG3 (as a function of time for exponential pulse loading) subjected to various numbers of layers and fiber orientations. It can be seen that the plate stiffness is directly proportional to the number of layers, in which the cross-ply contributes less stiffness than the angle-ply. The example demonstrates the dependence of the plate stiffness to the number of layers and fiber orientations, and hence an optimization problem for determining the optimal number of layers and fiber orientations should be applied to optimize the stiffness of the composite laminated plate.

\section{CONCLUSION}

In this paper, the cell-based smoothed discrete shear gap method (CS-FEM-DSG3) is extended to investigate the dynamic response of laminated composite plate under the effect of blast loading modeled by some trigonometric time functions. Numerical results demonstrate that the proposed method can achieve accurate results by using only a relative coarse mesh. Moreover, numerical examples also demonstrate the direct effect of the number of layers and the fiber orientation to the stiffness of plate, so an optimization algorithm should be applied to determine the optimal layer number and fiber orientations in the composite laminated problem. In addition, the new results of the numerical example which are used to demonstrate the effect of the layer's number and the fiber orientation to the dynamic response of the plate under the effect of blast loading can be served as reliable benchmark examples for later studies. The present CS-FEM-DSG3 is promising to extend to the problems with more complicated geometry domains and boundary conditions without existing available analytical solutions. 


\section{ACKNOWLEDGEMENTS}

This work was supported by Vietnam National Foundation for Science \& Technology Development (NAFOSTED), Ministry of Science \& Technology, under the basic research program (Project No.: 107.99-2014.11).

\section{REFERENCES}

[1] A. A. Khdeir and J. N. Reddy. Exact solutions for the transient response of symmetric crossply laminates using a higher-order plate theory. Composites Science and Technology, 34, (3), (1989), pp. 205-224.

[2] L. Librescu and A. Nosier. Response of laminated composite flat panels to sonic boom and explosive blast loadings. AIAA Journal, 28, (2), (1990), pp. 345-352.

[3] K. Y. Lam and L. Chun. Analysis of clamped laminated plates subjected to conventional blast. Composite Structures, 29, (3), (1994), pp. 311-321.

[4] C. Meimaris and J. D. Day. Dynamic response of laminated anisotropic plates. Computers $\mathcal{E}$ Structures, 55, (2), (1995), pp. 269-278.

[5] Y. V. Satish Kumar and M. Mukhopadhyay. Transient response analysis of laminated stiffened plates. Composite Structures, 58, (1), (2002), pp. 97-107.

[6] T. Hause and L. Librescu. Dynamic response of anisotropic sandwich flat panels to explosive pressure pulses. International Journal of Impact Engineering, 31, (5), (2005), pp. 607-628.

[7] L. Librescu, S.-Y. Oh, and J. Hohe. Dynamic response of anisotropic sandwich flat panels to underwater and in-air explosions. International Journal of Solids and Structures, 43, (13), (2006), pp. 3794-3816.

[8] G. R. Liu and T. T. Nguyen. Smoothed finite element methods. CRC Press, (2010).

[9] G. R. Liu, K. Y. Dai, and T. T. Nguyen. A smoothed finite element method for mechanics problems. Computational Mechanics, 39, (6), (2007), pp. 859-877.

[10] K.-U. Bletzinger, M. Bischoff, and E. Ramm. A unified approach for shear-locking-free triangular and rectangular shell finite elements. Computers $\mathcal{E}$ Structures, 75, (3), (2000), pp. 321334.

[11] T. Nguyen-Thoi, P. Phung-Van, H. Nguyen-Xuan, and C. Thai-Hoang. A cell-based smoothed discrete shear gap method using triangular elements for static and free vibration analyses of Reissner-Mindlin plates. International Journal for Numerical Methods in Engineering, 91, (7), (2012), pp. 705-741.

[12] T. Nguyen-Thoi, T. Bui-Xuan, P. Phung-Van, H. Nguyen-Xuan, and P. Ngo-Thanh. Static, free vibration and buckling analyses of stiffened plates by CS-FEM-DSG3 using triangular elements. Computers \& Structures, 125, (2013), pp. 100-113.

[13] T. Nguyen-Thoi, H. Luong-Van, P. Phung-Van, T. Rabczuk, and D. Tran-Trung. Dynamic responses of composite plates on the Pasternak foundation subjected to a moving mass by a cell-based smoothed discrete shear gap (CS-FEM-DSG3) method. International Journal of Composite Materials, 3, (2013), pp. 19-27.

[14] T. Nguyen-Thoi, P. Phung-Van, C. Thai-Hoang, and H. Nguyen-Xuan. A cell-based smoothed discrete shear gap method (CS-DSG3) using triangular elements for static and free vibration analyses of shell structures. International Journal of Mechanical Sciences, 74, (2013), pp. 32-45.

[15] P. Phung-Van, T. Nguyen-Thoi, H. Dang-Trung, and N. Nguyen-Minh. A cell-based smoothed discrete shear gap method (CS-FEM-DSG3) using layerwise theory based on the C0-HSDT for analyses of composite plates. Composite Structures, 111, (2014), pp. 553-565. 
[16] P. Phung-Van, T. Nguyen-Thoi, T. Le-Dinh, and H. Nguyen-Xuan. Static and free vibration analyses and dynamic control of composite plates integrated with piezoelectric sensors and actuators by the cell-based smoothed discrete shear gap method (CS-FEM-DSG3). Smart Materials and Structures, 22, (9), (2013). doi:10.1088/0964-1726/22/9/095026.

[17] R. D. Mindlin. Influence of rotary inertia and shear on flexural motions of isotropic elastic plates. Journal of Applied Mechanics, 18, (1951), pp. 31-38.

[18] E. Reissner. The effect of transverse shear deformation on the bending of elastic plates. Journal of Applied Mechanics, 12, (1945), pp. 69-77.

[19] J. N. Reddy. Mechanics of laminated composite plates and shells: Theory and analysis. CRC press, (2004).

[20] N. M. Newmark. A method of computation for structural dynamics. Journal of the Engineering Mechanics Division, 85, (3), (1959), pp. 67-94.

[21] S.-Y. Chang. Studies of Newmark method for solving nonlinear systems: (I) basic analysis. Journal of the Chinese Institute of Engineers, 27, (5), (2004), pp. 651-662. 